\title{
Fixed points for cyclic $R$-contractions and solution of nonlinear Volterra integro-differential equations
}

\author{
Mujahid Abbas ${ }^{1,2}$, Abdul Latif $^{2}$ and Yusuf I Suleiman ${ }^{3 *}$
}

\section{"Correspondence:}

yubram@yahoo.com

${ }^{3}$ Department of Mathematics, Kano

University of Science and

Technology, P.M.B. 3042, Wudil, Kano, Nigeria

Full list of author information is available at the end of the article

\begin{abstract}
In this paper, we introduce the notion of cyclic $R$-contraction mapping and then study the existence of fixed points for such mappings in the framework of metric spaces. Examples and application are presented to support the main result. Our result unify, complement, and generalize various comparable results in the existing literature.
\end{abstract}

MSC: $47 \mathrm{H} 10 ; 47 \mathrm{H} 09 ; 54 \mathrm{H} 25$

Keywords: fixed point; cyclic contractions; Meer-Keeler functions; simulation functions; R-contractions

\section{Introduction and preliminaries}

Let $(X, d)$ be any metric space, $Y$ a subset of $X$, and $f: X \rightarrow Y$. A point $x$ in $X$ that remains invariant under $f$ is called a fixed point of $f$. The set of all fixed points of $f$ is denoted by $F(f)$. A sequence $\left\{x_{n}\right\}$ in $X$ defined by $x_{n+1}=f\left(x_{n}\right)=f^{n}\left(x_{0}\right), n=0,1,2, \ldots$, is called a sequence of successive approximations of $f$ starting from $x_{0} \in X$. If it converges to a unique fixed point of $f$, then $f$ is called a Picard operator.

Fixed point theory plays a vital role in the study of existence of solutions of nonlinear problems arising in physical, biological, and social sciences. Some fixed point results simply ensure the existence of a solution but provide no information about the uniqueness and determination of the solution. The distinguishing feature of Banach-Caccioppoli contraction principle is that it addresses three most important aspects known as existence, uniqueness, and approximation or construction of a solution of linear and nonlinear problems. The simplicity and usefulness of this principle has motivated many researchers to extend it further, and hence there are a number of generalizations and modifications of the principle. One way to extend the Banach theorem is to weaken the contractive condition by employing the concept of comparison functions. For a detailed survey of such extensions obtained in this direction, we refer to $[1,2]$ and references therein.

We denote by $P_{c l}(X), \mathbb{N}, \mathbb{N}_{0}, \mathbb{R}$, and $\mathbb{R}^{+}$the collection of nonempty closed subsets of a metric space $(X, d)$, the set of positive integers, the set of nonnegative integers, the set of real numbers, and the set of positive real numbers, respectively. 
Let $(X, d)$ be a metric space. A self mapping $f$ on $X$ is called a $\varphi$-contraction if

$$
d(f x, f y) \leq \varphi(d(x, y))
$$

for all $x, y$ in $X$, where $\varphi$ is a suitable function on $[0, \infty)$, called a comparison function.

Definition 1.1 A map $\varphi_{1}:[0, \infty) \rightarrow[0, \infty)$ is said to be a Browder function if $\varphi_{1}$ is right continuous and monotone increasing.

Browder functions are examples of comparison functions. A self-mapping $f$ on $X$ is called a Browder contraction if

$$
d(f x, f y) \leq \varphi_{1}(d(x, y))
$$

for all $x, y \in X$, where $\varphi_{1}$ is a Browder function. Every Browder contraction on a complete metric space is a Picard operator [3]. Every Banach-contraction is a Browder contraction if $\varphi_{1}(t)=\gamma t$ for $\gamma \in[0,1)$.

Boyd and Wong [4] introduced a class of comparison functions as follows.

Definition 1.2 A function $\varphi_{2}:[0, \infty) \rightarrow[0, \infty)$ is called a Boyd-Wong function if $\varphi_{2}$ is upper semicontinuous from the right and $\varphi_{2}(t)<t$ for all $t>0$.

A self-mapping $f$ on $X$ is called a Boyd-Wong contraction if for all $x, y \in X$,

$$
d(f x, f y) \leq \varphi_{2}(d(x, y))
$$

where $\varphi_{2}$ is a Boyd-Wong function. Every Boyd-Wong contraction on a complete metric space is a Picard operator [4]. Note that Browder functions are Boyd-Wong functions.

Matkowski [5] initiated another class of comparison functions as follows.

Definition 1.3 A function $\phi:[0, \infty) \rightarrow[0, \infty)$ is called a Matkowski function if $\phi$ is increasing and $\lim _{n \rightarrow \infty} \phi^{n}(t)=0$ for all $t \geq 0$.

Every Matkowski function is a Boyd-Wond function ([1]).

Geraghty [6] defined the following class of comparison functions.

Let $\Phi$ be the class of all mappings $\beta:[0, \infty) \rightarrow[0,1)$ satisfying the condition: $\beta\left(t_{n}\right) \rightarrow 1$ implies $t_{n} \rightarrow 0$. Elements of $\Phi$ are called Geraghty functions.

Note that $\Phi \neq \phi$. For example, if a mapping $\beta:[0, \infty) \rightarrow[0,1)$ is defined by $\beta(x)=\frac{1}{1+x^{2}}$, $x \in[0, \infty)$, then $\beta \in \Phi$.

Let $(X, d)$ be a complete metric space, and $f: X \rightarrow X$. If there exists a Geraghty function $\beta$ such that for any $x, y \in X$, we have

$$
d(f x, f y) \leq \beta(d(x, y)) d(x, y)
$$

then $f$ is a Picard operator.

A self-mapping $f$ on $X$ is called a Meir-Keeler mapping if for any $\epsilon>0$, there exists $\delta_{\epsilon}>0$ such that for all $x, y \in X$ with $\epsilon \leq d(x, y)<\epsilon+\delta$, we have $d(f x, f y)<\epsilon$.

Lim [7] defined the notion of $L$-function to characterize the Meir-Keeler mappings. 
Definition 1.4 A mapping $\eta:[0, \infty) \rightarrow[0, \infty)$ is called a Lim function or L-function if $\eta(0)=0, \eta(t)>0$ for all $t>0$ and for any $\epsilon>0$, there exists $\delta_{\epsilon}>0$ such that $\eta(t) \leq \epsilon$ for all $t \in[\epsilon, \epsilon+\delta]$.

A self-map $f$ on a metric space $(X, d)$ is a Meir-Keeler mapping iff there exists an $L$ function $\eta$ such that $d(f x, f y)<\eta(d(x, y))$ for all $x, y \in X$ with $d(x, y)>0$.

The notion of simulation functions was introduced by Khojasteh et al. [8] and then modified in [9] and [10].

Definition 1.5 A mapping $\zeta:[0, \infty) \times[0, \infty) \rightarrow \mathbb{R}$ is called a simulation function if the following conditions hold:

$\left(\zeta_{1}\right) \zeta(t, s)<s-t$ for all $t, s>0$;

$\left(\zeta_{2}\right)$ if $\left\{t_{n}\right\}$ and $\left\{s_{n}\right\}$ are sequences in $(0, \infty)$ such that $\lim _{n \rightarrow \infty} t_{n}=\lim _{n \rightarrow \infty} s_{n} \in(0, \infty)$ and $t_{n}<s_{n}$ for all $n \in \mathbb{N}$ then $\limsup _{n \rightarrow \infty} \zeta\left(t_{n}, s_{n}\right)<0$.

Note that Boyd-Wong functions are simulation functions.

Consistent with Rodan-Lopez-de-Hierro and Shahzad [10], the following definitions, examples, and results will be needed in the sequel.

Definition 1.6 Let $A \subset \mathbb{R}$ be a nonempty set. A function $\varrho: A \times A \rightarrow \mathbb{R}$ is called an $R$ function if:

$\left(\varrho_{1}\right)$ for any sequence $\left\{a_{n}\right\} \subset(0, \infty) \cap A$ with $\varrho\left(a_{n+1}, a_{n}\right)>0 \forall n \in \mathbb{N}$, we have $\lim _{n \rightarrow \infty} a_{n}=$ 0 ;

$\left(\varrho_{2}\right)$ for any sequences $\left\{a_{n}\right\},\left\{b_{n}\right\}$ in $(0, \infty) \cap A$ satisfying $\varrho\left(a_{n}, b_{n}\right)>0 \forall n \in \mathbb{N}, \lim _{n \rightarrow \infty} a_{n}=$ $\lim _{n \rightarrow \infty} b_{n}=L \geq 0$ and $L<a_{n}$ imply that $L=0$.

Example 1.7 ([10], Example 18) Define $\varrho:[0, \infty) \times[0, \infty) \rightarrow \mathbb{R}$ by

$$
\varrho(t, s)= \begin{cases}\frac{1}{2} s-t & \text { if } t<s \\ 0 & \text { if } t \geq s\end{cases}
$$

Then $\varrho$ is an $R$-function that is not a simulation function.

Rodan-Lopez-de-Hierro and Shahzad [10] also considered the following condition:

$\left(\varrho_{3}\right)$ If $\left\{a_{n}\right\}$ and $\left\{b_{n}\right\}$ are sequences in $(0, \infty) \cap A$ such that $\lim _{n \rightarrow \infty} b_{n}=0$ and $\varrho\left(a_{n}, b_{n}\right)>0$ $\forall n \in \mathbb{N}$, then $\lim _{n \rightarrow \infty} a_{n}=0$.

Example 1.8 ([10], Lemma 15) Every simulation function is an $R$-function that satisfies $\left(\varrho_{3}\right)$.

Example 1.9 ([10]) If $\phi:[0, \infty) \rightarrow[0,1)$ is a Geraghty function, then $\varrho_{\phi}:[0, \infty) \times$ $[0, \infty) \rightarrow \mathbb{R}$ defined by

$$
\varrho_{\phi}(t, s)=\phi(s) s-t
$$

is an $R$-function satisfying $\left(\varrho_{3}\right)$. 
Example $1.10([10])$ If $\phi:[0, \infty) \rightarrow[0, \infty)$ is an L-function, then $\varrho_{\phi}:[0, \infty) \times[0, \infty) \rightarrow \mathbb{R}$ defined by $\varrho_{\phi}(t, s)=\phi(s)-t$ is an $R$-function satisfying $\left(\varrho_{3}\right)$.

Definition 1.11 Let $(X, d)$ be a metric space. A self-map $f$ of $X$ is called an $R$-contraction if there exists $\varrho \in R_{A}$ such that $\operatorname{ran}(d) \subseteq A$ and $\varrho(d(f x, f y), d(x, y))>0$ for all $x, y \in X$ with $x \neq y$, where $R_{A}$ is the family of all functions $\varrho: A \times A \rightarrow \mathbb{R}$ satisfying the conditions $\left(\varrho_{1}\right)$ and $\left(\varrho_{2}\right)$, and $\operatorname{ran}(d)$ is the range of the metric $d$ defined by $\operatorname{ran}(d)=\{d(x, y): x, y \in X\} \subseteq[0, \infty)$.

Definition 1.12 Let $X$ be a nonempty set, $p$ a positive integer, and $f$ a self-map on $X$. If $\left\{B_{i}: i=1,2, \ldots, p\right\}$ is a finite family of nonempty subsets of $X$ such that $f\left(B_{1}\right) \subset B_{2}, f\left(B_{2}\right) \subset$ $B_{3}, \ldots, f\left(B_{p-1}\right) \subset B_{p}, f\left(B_{p}\right) \subset B_{1}$. Then the set $\bigcup_{i=1}^{p} B_{i}$ is called a cyclic representation of $X$ with respect to $f$.

Kirk et al. [11] introduced the notion of cyclic $\varphi$-contraction mappings as follows.

Definition 1.13 Let $(X, d)$ be a metric space, and $\left\{B_{i}: i=1,2, \ldots, p\right\}$ be a finite family of nonempty closed subsets of $X$. An operator $f: \bigcup_{i=1}^{p} B_{i} \rightarrow \bigcup_{i=1}^{p} B_{i}$ is said to be a cyclic $\varphi$ contraction if $\bigcup_{i=1}^{p} B_{i}$ is a cyclic representation of $X$ with respect to $f$ and

$$
d(f x, f y) \leq \varphi(d(x, y))
$$

for all $x \in B_{i}, y \in B_{i+1}, 1 \leq i \leq p$, where $B_{p+1}=B_{1}$, and $\varphi$ is a Boyd-Wong function.

Kirk et al. [11] established the following fixed point results for Geraghty, Boyd-Wong, and Caristi cyclic $\varphi$-contractions.

Theorem 1.14 Let $(X, d)$ be a complete metric space, and p a natural number. Suppose that a self-mapping $f$ is a cyclic $\varphi$-contraction on $\bigcup_{i=1}^{p} B_{i}$. Then there exists a unique element $z \in \bigcap_{i=1}^{p} B_{i}$ such that $f(z)=z$.

Later, Pacurar and Rus [12] introduced the notion of weakly cyclic $\varphi$-contraction. Karapinar [13] improved the results in [12] dropping the requirement of continuity. For more results in this direction, we refer to [14-16] and references therein.

We now introduce the following notion of cyclic R-contraction mapping.

Definition 1.15 Let $(X, d)$ be a metric space, and $B_{1}, B_{2}, \ldots, B_{p} \in P_{c l}(X)$. A mapping $f$ : $\bigcup_{i=1}^{p} B_{i} \rightarrow \bigcup_{i=1}^{p} B_{i}$ is said to be a cyclic $R$-contraction if

(i) there exists $\varrho \in R_{A}$ with $\operatorname{ran}(d) \subseteq A$;

(ii) $\bigcup_{i=1}^{p} B_{i}$ is a cyclic representation of $X$ with respect to $f$, and

(iii) $\varrho(d(f x, f y), d(x, y))>0$ for all $x \in B_{i}, y \in B_{i+1}, 1 \leq i \leq p$, where $B_{p+1}=B_{1}$.

Meir-Keeler, Geraghty, and simulation contractions are typical examples of $R$-contractions that satisfy $\left(\varrho_{3}\right)$. Consequently, the cyclic- $R$-contractions are a generalization of cyclic Meir-Keeler, cyclic Geraghty, cyclic manageable, and cyclic simulative contractions.

In this paper, we prove a fixed point result for cyclic $R$-contractions. Our result extends and unifies fixed point results involving Boyd-Wong cyclic contractions, Meir-keeler cyclic contractions, and Geraghty cyclic contraction mappings. Applying our result, we obtain the existence of solutions of nonlinear Volterra integro differential equations. 


\section{Main results}

We start with the following result.

Theorem 2.1 Let $(X, d)$ be a complete metric space, and $B_{1}, B_{2}, \ldots, B_{p} \in P_{c l}(X)$. Suppose that a mapping $f$ is a cyclic $R$-contraction on $\bigcup_{i=1}^{p} B_{i}$. Then there exists a unique element $z \in \bigcap_{i=1}^{p} B_{i}$ such that $f(z)=z$.

Proof Let $x_{0}$ be a given point in $\bigcup_{i=1}^{p} B_{i}$. Then there exists $i_{0}$ in $\{1,2, \ldots, p\}$ such that $x_{0} \in B_{i_{0}}$. Since $f\left(B_{i_{0}}\right) \subset B_{i_{0}+1}$, we have that $f\left(x_{0}\right) \in B_{i_{0}+1}$. Thus, there exists $x_{1} \in B_{i_{0}+1}$ with $f\left(x_{0}\right)=x_{1}$. Similarly, there exists $x_{2} \in B_{i_{0}+2}$ with $f\left(x_{1}\right)=x_{2}$. Continuing in this way, we can construct a sequence in $\bigcup_{i=1}^{p} B_{i}$ by $x_{n}=f\left(x_{n-1}\right)=f^{n}\left(x_{0}\right) \in B_{i_{0}+n}$ for all $n \in \mathbb{N}$. Now, if $x_{n+1}=x_{n}$ for some $n \in \mathbb{N}$, then the result follows immediately. Suppose that $x_{n+1} \neq x_{n}$ for all $n \in \mathbb{N}$. Note that

$$
\varrho\left(d\left(f x_{n-1}, f x_{n}\right), d\left(x_{n-1}, x_{n}\right)\right)=\varrho\left(d\left(x_{n}, x_{n+1}\right), d\left(x_{n-1}, x_{n}\right)\right)>0 \quad \text { for all } n \in \mathbb{N} \text {. }
$$

From property $\left(\varrho_{1}\right)$ of an $R$-function we have

$$
\lim _{n \rightarrow \infty} d\left(x_{n}, x_{n+1}\right)=0
$$

We now show that $\left\{x_{n}\right\}$ is a Cauchy sequence. If not, then there exists $L>0$ such that for any $k \in \mathbb{N}$, we can construct two subsequences $\left\{x_{m_{k}}\right\}$ and $\left\{x_{n_{k}}\right\}$ of $\left\{x_{n}\right\}$ with $n_{k}>m_{k} \geq k$ satisfying

$$
d\left(x_{m_{k}}, x_{n_{k}}\right)>L
$$

Without any loss of generality, we assume that $n_{k}$ is the smallest integer greater than $m_{k}$ for which the last inequality holds. We can choose $j_{k} \in\{1,2, \ldots, p\}$ such that $n_{k}>m_{k}>$ $m_{k}-j_{k}$ with $n_{k}$ belonging to the residue class of $m_{k}-j_{k}+1$, and hence $x_{m_{k}-j_{k}}$ and $x_{n_{k}}$ lie in different adjacently labeled sets $B_{i}$ and $B_{i+1}$ for some $i \in\{1,2, \ldots, p\}$. Thus,

$$
d\left(x_{m_{k}-j_{k}}, x_{n_{k}}\right)>L \quad \text { and } \quad d\left(x_{m_{k}-j_{k}}, x_{n_{k}-2}\right) \leq L \quad \text { for all } k \in \mathbb{N}
$$

By (2.2) we have

$$
\begin{aligned}
L & <d\left(x_{m_{k}-j_{k}}, x_{n_{k}}\right) \\
& <d\left(x_{m_{k}-j_{k}}, x_{n_{k}-2}\right)+d\left(x_{n_{k}-2}, x_{n_{k}-1}\right)+d\left(x_{n_{k}-1}, x_{n_{k}}\right) \\
& \leq L+d\left(x_{n_{k}-2}, x_{n_{k}-1}\right)+d\left(x_{n_{k}-1}, x_{n_{k}}\right) .
\end{aligned}
$$

Taking the limit as $k \rightarrow \infty$ on both sides of this inequality, we have

$$
\lim _{k \rightarrow \infty} d\left(x_{m_{k}-j_{k}}, x_{n_{k}}\right)=L
$$

Similarly,

$$
\begin{aligned}
L & <d\left(x_{m_{k}-j_{k}}, x_{n_{k}}\right) \\
& <d\left(x_{m_{k}-j_{k}}, x_{m_{k}-j_{k}-1}\right)+d\left(x_{m_{k}-j_{k}-1}, x_{n_{k}-1}\right)+d\left(x_{n_{k}-1}, x_{n_{k}}\right) .
\end{aligned}
$$


Also,

$$
d\left(x_{m_{k}-j_{k}-1}, x_{n_{k}-1}\right) \leq d\left(x_{m_{k}-j_{k}-1}, x_{m_{k}-j_{k}}\right)+d\left(x_{m_{k}-j_{k}}, x_{n_{k}}\right)+d\left(x_{n_{k}}, x_{n_{k}-1}\right)
$$

Taking the limit as $k \rightarrow \infty$ on both sides of (2.5) and (2.6), we obtain that

$$
\lim _{k \rightarrow \infty} d\left(x_{m_{k}-j_{k}-1}, x_{n_{k}-1}\right)=L
$$

Now, since

$$
\begin{aligned}
& d\left(x_{m_{k}-j_{k}}, x_{n_{k}}\right)>L \quad \text { for all } k \in \mathbb{N}, \\
& \lim _{k \rightarrow \infty} d\left(x_{m_{k}-j_{k}-1}, x_{n_{k}-1}\right)=\lim _{k \rightarrow \infty} d\left(x_{m_{k}-j_{k}}, x_{n_{k}}\right)=L, \quad \text { and } \\
& \varrho\left(d\left(f x_{m_{k}-j_{k}-1}, f x_{n_{k}-1}\right), d\left(x_{m_{k}-j_{k}-1}, x_{n_{k}-1}\right)\right)=\varrho\left(d\left(x_{m_{k}-j_{k}}, x_{n_{k}}\right), d\left(x_{m_{k}-j_{k}-1}, x_{n_{k}-1}\right)\right)>0,
\end{aligned}
$$

then by property $\left(\varrho_{2}\right)$ of an $R$-function, we conclude that $0=L>0$, a contradiction. Hence, $\left\{x_{n}\right\}$ is a Cauchy sequence in $X$. Since $(X, d)$ is complete, there exists $\gamma \in X$ such that $\lim _{n \rightarrow \infty} x_{n}=\gamma$. Since $\bigcup_{i=1}^{p} B_{i}$ is a cyclic representation of $X$ with respect to $f$, there exist subsequences $\left\{x_{n_{p}}\right\},\left\{x_{n_{p+1}}\right\},\left\{x_{n_{p+2}}\right\}, \ldots,\left\{x_{n_{p+p-2}}\right\},\left\{x_{n_{p+p-1}}\right\}$, and $\left\{x_{n_{p+p}}\right\}$ of $\left\{x_{n}\right\}$ such that $\left\{x_{n_{p}}\right\} \subset B_{1},\left\{x_{n_{p+1}}\right\} \subset B_{2},\left\{x_{n_{p+2}}\right\} \subset B_{3}, \ldots,\left\{x_{n_{p+p-2}}\right\} \subset B_{p-1},\left\{x_{n_{p+p-1}}\right\} \subset B_{p}$, and $\left\{x_{n_{p+p}}\right\} \subset$ $B_{p+1}=B_{1}$. Since each $B_{i}, i \in\{1,2,3, \ldots, p\}$, is a closed subset of $X$ and $\lim _{n \rightarrow \infty} x_{n}=\gamma$, we deduce that $\gamma \in \bigcap_{i=1}^{p} B_{i}$.

Note that for each $n \in \mathbb{N}$, there exists $i_{n} \in\{1,2, \ldots, p\}$ such that $x_{n-1} \in B_{i_{n-1}}, x_{n} \in B_{i_{n}}$, and $\gamma \in B_{i_{n}}$. Thus,

$$
\varrho\left(d\left(f \gamma, f x_{n-1}\right), d\left(\gamma, x_{n-1}\right)\right)=\varrho\left(d\left(f \gamma, x_{n}\right), d\left(\gamma, x_{n-1}\right)\right)>0 \quad \text { for all } n \in \mathbb{N} .
$$

Using property $\left(\varrho_{1}\right)$ of an $R$-function, we obtain that $\lim _{n \rightarrow \infty} d\left(f \gamma, x_{n}\right)=d(f \gamma, \gamma)=0$.

Therefore, $\gamma$ is a fixed point of $f$ in $\bigcap_{i=1}^{p} B_{i}$.

Uniqueness: Suppose that there exists another fixed point $x^{*}$ of $f$ in $\bigcap_{i=1}^{p} B_{i}$, that is, $d\left(x^{*}, \gamma\right)>0$ and $d\left(f \gamma, f x^{*}\right)=d\left(\gamma, x^{*}\right)$. Since $f$ is a cyclic $R$-contraction, we have

$$
\varrho\left(d\left(f \gamma, f x^{*}\right), d\left(\gamma, x^{*}\right)\right)>0 .
$$

By property $\left(\varrho_{1}\right)$ of an $R$-function we have $0<d\left(x^{*}, \gamma\right)=\lim _{n \rightarrow \infty} d\left(x^{*}, \gamma\right)=0$, a contradiction. This establishes the result.

Example 2.2 Let $X=\mathbb{R}$ be endowed with the Euclidean metric $d(x, y)=|x-y|$ for all $x, y \in X$. Suppose that $B_{1}=[-1,0], B_{2}=[0,1]$, and $A=\operatorname{ran}(d) \subset[0, \infty)$. Define $f: \bigcup_{i=1}^{2} B_{i} \rightarrow$ $\bigcup_{i=1}^{2} B_{i}$ and $\varrho: A \times A \rightarrow \mathbb{R}$ as

$$
f(x)=-\frac{x}{5} \quad \text { and } \quad \varrho(t, s)= \begin{cases}\frac{1}{2} s-t & \text { if } t<s \\ 0 & \text { if } t \geq s .\end{cases}
$$

Note that $(X, d)$ is a complete space and $B_{1}$ and $B_{2}$ are closed in $X$. If $x \in B_{1}$, that is, $-1 \leq$ $x \leq 0$, then $0 \leq-\frac{x}{5} \leq \frac{1}{5}$ implies that $f(x) \in B_{2}$. Similarly, if $x \in B_{2}$, that is, $0 \leq x \leq 1$, then $-\frac{1}{5} \leq-\frac{x}{5} \leq 0$ implies that $f(x) \in B_{1}$. 
Further, $\varrho(d(f x, f y), d(x, y))=\frac{1}{2} d(x, y)-d(f x, f y)=\frac{3}{10}|x-y|>0$ for all $x \in B_{1}, y \in B_{2}$. Thus, all conditions of Theorem 2.1 are satisfied. Moreover, $z=0 \in \bigcap_{i=1}^{2} B_{i}$ is a fixed point of $f$.

Example 2.3 Let $X=\mathbb{R}$ and $d(x, y)=|x-y|$ for all $x, y \in X$. Suppose that $B_{1}=\left\{\frac{1}{2 n}\right\}_{n \in \mathbb{N} \cup\{0\}}$, $B_{2}=\left\{-\frac{1}{2 n-1}\right\}_{n \in \mathbb{N} \cup\{0\}}$, and $A=\operatorname{ran}(d) \subset[0, \infty)$. Define $f: \bigcup_{i=1}^{2} B_{i} \rightarrow \bigcup_{i=1}^{2} B_{i}$ and $\varrho: A \times A \rightarrow$ $\mathbb{R}$ as

$$
f(x)=\left\{\begin{array}{ll}
-\frac{x}{4} & \text { if } x \in B_{1}, \\
-\frac{x}{5} & \text { if } x \in B_{2},
\end{array} \text { and } \quad \varrho(t, s)= \begin{cases}\frac{1}{2} s-t & \text { if } t<s \\
0 & \text { if } t \geq s .\end{cases}\right.
$$

It is clear that $B_{1}$ and $B_{2}$ are closed subsets of a complete metric space $(X, d)$ such that $f\left(B_{1}\right) \subset B_{2}$ and $f\left(B_{2}\right) \subset B_{1}$. Note that

$$
\begin{aligned}
\varrho(d(f x, f y), d(x, y)) & =\frac{1}{2} d(x, y)-d(f x, f y) \\
& =\frac{1}{2}|x-y|-\left|\frac{x}{4}-\frac{y}{5}\right| \\
& >\frac{1}{2}|x-y|-\left|\frac{x}{4}-\frac{x}{20}-\frac{y}{5}\right| \\
& =\frac{3}{10}|x-y|>0
\end{aligned}
$$

for all $x \in B_{1}, y \in B_{2}$. Hence, all conditions of Theorem 2.1 are satisfied, and $z=0 \in \bigcap_{i=1}^{2} B_{i}$ is a fixed point of $f$.

Remark 2.4 In this example, the mapping is a cyclic $R$-contraction that is neither a MeirKeeler cyclic contraction nor a simulative cyclic contraction and hence neither a BoydWong nor a Geraghty cyclic contraction. Indeed, if we take $t=s=1$, then $\left(\zeta_{2}\right)$ fails.

Corollary 2.5 Let $(X, d)$ be a complete metric space, and $B_{1}, B_{2}, \ldots, B_{p} \in P_{c l}(X)$. Suppose that a mapping $f$ is a manageable cyclic contraction, or a simulative cyclic contraction, or a Geraghty cyclic contraction, or a Boyd-Wong cyclic contraction, or a Meir-Keeler cyclic contraction on $\bigcup_{i=1}^{p} B_{i}$. Then there exists a unique element $z \in \bigcap_{i=1}^{p} B_{i}$ such that $f(z)=z$.

\section{Application to nonlinear Volterra integral equations}

Motivated by the work in [17], we obtain the existence and uniqueness of solutions for nonlinear Volterra integral differential equations.

Consider the following problem:

$$
u(x, y)=f(x, y)+\int_{0}^{x} g(x, y, \xi, u(\xi, y)) d \xi+\int_{0}^{x} \int_{0}^{y} h(x, y, \sigma, \tau, u(\sigma, \tau)) d \tau d \sigma
$$

where $f \in C\left(\mathbb{R}^{+} \times \mathbb{R}^{+}, \mathbb{R}\right), g \in C\left(E_{1} \times \mathbb{R}^{+}, \mathbb{R}\right), h \in C\left(E_{2} \times \mathbb{R}^{+}, \mathbb{R}\right), E_{1}=\{f(x, y, s): s \leq x \in$ $[0, \infty), y \in[0, \infty)\}$, and $E_{2}=\{f(x, y, s, t): s \leq x \in[0, \infty), t \leq y \in[0, \infty)\}$.

Let $X$ be the space of functions $z \in C\left(\mathbb{R}^{+} \times \mathbb{R}^{+}, \mathbb{R}\right)$ satisfying $|z(x, t)|=O\left(e^{\lambda(x+y)}\right)$, where $\lambda$ is a positive constant, that is, $|z(x, y)| \leq M_{0} e^{\lambda(x+y)}$ for some constant $M_{0}>0$.

Define the norm on $X$ by $\|z\|_{X}=\sup _{(x, y) \in\left(\mathbb{R}^{+} \times \mathbb{R}^{+}\right)}\left\{|z(x, y)| e^{-\lambda(x+y)}\right\}$. 
Note that $\left(X,\|\cdot\|_{X}\right)$ is a Banach space. Define the mapping $T: X \rightarrow X$ by

$$
T(u(x, y))=f(x, y)+\int_{0}^{x} g(x, y, \xi, u(\xi, y)) d \xi+\int_{0}^{x} \int_{0}^{y} h(x, y, \sigma, \tau, u(\sigma, \tau)) d \tau d \sigma
$$

for every $u \in X$. It is easy to see that $u^{*} \in X$ is a solution of problem (3.1) if $T\left(u^{*}\right)=u^{*}$.

Theorem 3.1 Suppose that problem (3.1) satisfies the following conditions:

(I)

$$
|g(x, y, \xi, u)-g(x, y, \xi, \bar{u})| \leq h_{1}(x, y, \xi)|u-\bar{u}|
$$

and

$$
|h(x, y, \sigma, \tau, u)-h(x, y, \sigma, \tau, \bar{u})| \leq h_{2}(x, y, \sigma, \tau)|u-\bar{u}|
$$

where $h_{1} \in C\left(E_{1},[0, \infty)\right)$ and $h_{2} \in C\left(E_{2},[0, \infty)\right)$;

(II) There exist $\alpha, \beta$ in $X$ and $\alpha_{0}, \beta_{0}$ in $\mathbb{R}$ with $\alpha_{0} \leq \alpha(x, t) \leq \beta(x, t) \leq \beta_{0}(x, t)$ such that

$$
\alpha(x, t) \leq f(x, t)+\int_{0}^{x} g(t, s, \xi, \beta(\xi, s)) d \xi+\int_{0}^{x} \int_{0}^{y} h(t, s, \sigma, \tau, \beta(\sigma, \tau)) d \tau d \sigma
$$

and

$$
\beta(x, t) \geq f(x, t)+\int_{0}^{x} g(t, s, \xi, \alpha(\xi, s)) d \xi+\int_{0}^{x} \int_{0}^{y} h(t, s, \sigma, \tau, \alpha(\sigma, \tau)) d \tau d \sigma
$$

for all $x, t \in[0, \infty)$

(III)

$$
\int_{0}^{x} h_{1}(x, y, \xi) e^{\lambda(x+y)} d \xi+\int_{0}^{x} \int_{0}^{y} h_{2}(x, y, \sigma, \tau) e^{\lambda(\sigma+\tau)} d \tau d \sigma \leq \delta_{1} e^{\lambda(x+y)}
$$

and

$$
\left|f(x, t)+\int_{0}^{x} g(x, y, \xi, 0) d \xi+\int_{0}^{x} \int_{0}^{y} h(x, y, \sigma, \tau, 0) d \tau d \sigma\right| \leq \delta_{2} e^{\lambda(x+y)}
$$

for some nonnegative constants $\delta_{1}, \delta_{2}<1$;

(IV) There exist $\alpha, \beta$ in $X$ such that $\alpha(t) \leq \beta(t), T(\alpha(x, t)) \leq \beta(x, t)$, and $T(\beta(x, t)) \geq \alpha(x, t)$. Then the integral Eq. (3.1) has a unique solution $u^{*}$ in $\varpi=\{u \in X: \alpha(x, y) \leq u(x, y) \leq \beta(x, y)\}$.

Proof Let $B_{1}=\{u \in X: u(x, t) \leq \beta(x, t)\}$ and $B_{2}=\{u \in X: u(x, t) \geq \alpha(x, t)\}$. Then $B_{1}$ and $B_{2}$ are closed subsets of the complete metric space $X$. If $u \in B_{1}$, then by conditions (I), (II), and (IV) we conclude that $T(u(x, t)) \geq \alpha(x, t)$. Hence, $T u \in B_{2}$. Similarly, $u \in B_{2}$ implies that $T u \in B_{1}$, and hence $T\left(B_{1}\right) \subset B_{2}$ and $T\left(B_{2}\right) \subset B_{1}$.

If $u \in B_{1}$ and $v \in B_{2}$, then $u(x, t) \leq \beta(x, t) \leq \beta_{0}$ and $v(x, t) \geq \alpha(x, t) \geq \alpha_{0}$. From conditions (I) and (III) we obtain that

$$
\|T(u(x, y))-T(v(x, y))\|_{X} \leq \delta_{1}\|u-v\|_{X} e^{\lambda(x+y)}
$$


Thus,

$$
\|T u-T v\|_{X} \leq \varsigma\|u-v\|_{X} e^{\lambda(x+y)}, \quad \text { where } \varsigma=\delta_{1}<1
$$

Taking $\varrho(t, s)=\varsigma s-t$, we have

$$
\varrho\left(\|T u-T v\|_{X},\|u-v\|_{X}\right)=\varsigma\|u-v\|_{X}-\|T u-T v\|_{X}>0, \quad u \neq v .
$$

Consequently, $T$ is a cyclic $R$-contraction on $\bigcup_{i=1}^{2} B_{i}$. By Theorem 2.1, $T$ has a unique fixed point $u^{*}$ in $\bigcap_{i=1}^{2} B_{i} \in \varpi$, which is the solution of the integral-differential Eq. (3.1).

\section{Competing interests}

The authors declare that they have no competing interests.

\section{Authors' contributions}

All authors contributed equally and significantly in writing this paper. The authors declare that they have no competing interests. All authors read and approved the final manuscript.

\section{Author details}

${ }^{1}$ Department of Mathematics and Applied Mathematics, University of Pretoria, Hatfield, South Africa. ${ }^{2}$ Department of Mathematics, King Abdulaziz University, P.O. Box 80203, Jeddah, 21589, Saudi Arabia. ${ }^{3}$ Department of Mathematics, Kano University of Science and Technology, P.M.B. 3042, Wudil, Kano, Nigeria.

\section{Acknowledgements}

The authors are very indebted to both reviewers for a number of useful suggestions to improve this paper.

\section{Received: 16 December 2015 Accepted: 4 May 2016 Published online: 11 May 2016}

\section{References}

1. Berinde, V: Iterative Approximation of Fixed Points. Springer, Berlin (2007)

2. Latif, A: Banach contraction principle and its generalizations. In: Almezel, S, Ansari, QH, Khamsi, MA (eds.) Monograph: Topics in Fixed Point Theory, pp. 33-64. Springer, Berlin (2014)

3. Browder, F: On the convergence of successive approximations for nonlinear functional equations. Indag. Math. 30, 27-35 (1968)

4. Boyd, DW, Wong, JSW: On nonlinear contractions. Proc. Am. Math. Soc. 20, $458-464$ (1969)

5. Matkowski, J: Integrable solutions of functional equations. Diss. Math. 127, 1-68 (1975)

6. Geraghty, M: On contractive mappings. Proc. Am. Math. Soc. 40, 604-608 (1973)

7. Lim, TC: On characterizations of Meir-Keeler contractive maps. Nonlinear Anal. 46, 113-120 (2001)

8. Khojasteh, F, Shukla, S, Radenović, S: A new approach to the study of fixed point theorems via simulation functions. Filomat 29(6), 1189-1194 (2015)

9. Argoubi, H, Samet, B, Vetro, C: Nonlinear contractions involving simulation functions in a metric space with a partial order. J. Nonlinear Sci. Appl. 8(6), 1082-1094 (2015)

10. Roldan-Lopez-de-Hierro, AF, Shahzad, N: New fixed point theorem under R-contractions. Fixed Point Theory Appl. 2015, 98 (2015)

11. Kirk, WA, Srinivasan, PS, Veeramani, P: Fixed points for mappings satisfying cyclical contractive conditions. Fixed Point Theory 4(1), 79-89 (2003)

12. Păcurar, M, Rus, IA: Fixed point theory for cyclic $\phi$-contractions. Nonlinear Anal., Theory Methods Appl. 72(3-4), 1181-1187 (2010)

13. Karapinar, E: Fixed point theory for cyclic weak $\phi$-contraction. Appl. Math. Lett. 24(6), $822-825$ (2011)

14. Abbas, M, Nazir, T, Romaguera, $S$ : Fixed point results for generalized cyclic contraction mappings in partial metric spaces. Rev. R. Acad. Cienc. Exactas Fís. Nat., Ser. A Mat. 106, 287-297 (2012)

15. Karapinar, E, Moradi, S: Fixed point theory for cyclic generalized $(\varphi-\phi)$-contraction mappings. Ann. Univ. Ferrara 59, 117-125 (2013)

16. Karapinar, E: Best proximity points of cyclic mappings. Appl. Math. Lett. 25(11), 1761-1766 (2012)

17. Nashine, HK, Pathak, RP, Somvanshi, PS, Pantelic, S, Kumam, P: Solutions for a class of nonlinear Volterra integral and integro-differential equation using cyclic $(\varphi, \psi, \theta)$-contraction. Adv. Differ. Equ. 2013, 106 (2013) 